\title{
Diseño de una intervención basada en técnicas de regulación emocional y psicología positiva para un caso de trastorno de depresión mayor y rasgos de personalidad límite
}

\author{
ANDREa Delgado CARRETERo \\ al350090@uji.es \\ Berenice SeRrano ZÁrate \\ bserrano@uji.es
}

\section{Resumen}

La depresión está muy asociada con sintomatología en personas con trastorno límite de la personalidad (TLP), se estima que el $32 \%$ de pacientes con TLP padecen un trastorno de depresión mayor (TDM). Por otra parte, se ha evidenciado la eficacia de intervenciones basadas en regulación emocional y psicología positiva para la reducción de la sintomatología depresiva. Objetivo: Diseñar una intervención para un caso diagnosticado con TDM y rasgos de TLP, basada en técnicas de regulación emocional y psicología positiva. Método: La intervención se llevó a cabo con una mujer de 23 años quien experimentaba sintomatología depresiva, dificultades de regulación emocional, y rasgos límite de la personalidad. Se ha diseñado un protocolo de evaluación el cual mide antes y después de la intervención el estado de ánimo, autoestima, ansiedad, síntomas de trastorno de personalidad, regulación emocional, expectativas y opinión del tratamiento, alianza terapéutica y gravedad valorada por el terapeuta. Asimismo, se evaluó semanalmente a través de registros específicos para este estudio. También se ha diseñado un protocolo de intervención, integrado por: psicoeducación, toma de conciencia, regulación emocional, psicología positiva, y prevención de recaídas. La intervención se realizó durante 13 sesiones con una duración de 60 min., con periodicidad semanal. Resultados: Se produjo una diminución de la sintomatología depresiva y ansiosa, un incremento de la autoestima, y mejoras en la regulación emocional. Conclusiones: El uso de técnicas como la regulación emocional y la psicología positiva, podrían resultar de gran utilidad en el tratamiento del TDM en comorbilidad con problemas de la personalidad.

Palabras clave: depresión, trastorno límite de personalidad, regulación emocional, psicología positiva, autoestima.

\section{Abstract}

Depression are highly associated with Borderline Personality Disorder (BPD) symptoms, being estimated that a $32 \%$ of BPD patients suffer major depressive disorder 
(MDD). Furthermore, interventions based on emotional regulation and positive psychology have shown their effectiveness in the depressive symptomatology reduction. Purpose: To design an intervention based on emotional regulation techniques and positive psychology for a case diagnosed with MDD and BPD traits. Methods: The intervention is applied to a 23-year-old woman who experiences depressive symptomatology, difficulties in emotional regulation, and BPD traits. An evaluation protocol for mood, selfesteem, anxiety and personality disorder symptoms, emotional regulation, expectative and treatment opinion, therapeutic alliance and severity evaluated by the therapist was designed and applied previous and after the intervention, including a weekly evaluation through specific registers designed for this studio. The designed intervention protocol was composed by psychoeducation, consciousness, emotional regulation, positive psychology, and relapse prevention. The intervention was applied through 60 minutes weekly sessions, during an intervention period of 13 sessions. Results: Clinical significant changes in patient symptomatology were observed, showing anxiety and depressive symptomatology reduction, self- esteem increment and improvement of emotional regulation. Conclusion: The use of techniques such as emotional regulation and positive psychology could be very useful in the treatment of MDD in comorbidity with personality problems.

Keywords: depression, borderline personality disorder, emotional regulation, positive psychology, self-esteem.

\section{Introducción}

El estado de ánimo sufre oscilaciones a lo largo del tiempo y presenta una estrecha relación con la personalidad. Personas con determinados rasgos de personalidad, son más vulnerables a experimentar un estado de ánimo u otro (Hervás \& Vázquez, 2006). Asimismo, circunstancias vividas, ya sean aquellas que favorecen el desarrollo o lo dificultan, suponen una repercusión en nuestro ajuste emocional. Se ha estudiado que las dificultades vitales presentan una estrecha relación con la ocurrencia de sintomatología depresiva, características de personalidad desadaptativas y dificultades en la regulación emocional. A menudo los episodios de sintomatología depresiva acontecen tras un estrés psicosocial grave, por lo que se estima que desempeñan un papel importante en la precipitación del primer episodio (Lloyd, Klinteberg \& DeMarinis, 2016). En la misma línea, ambientes muy invalidantes a nivel familiar (problemas en la interacción y el apego, experiencias traumáticas o negligencias emocionales) suponen factores de riesgo para el desarrollo de rasgos desadaptativos o trastornos de personalidad. Todo ello influye en nuestro desarrollo personal, sistema emocional, autoestima, regulación afectiva, capacidad de afrontamiento y resolución de problemas y por lo tanto en nuestro estado de ánimo. Dicha sintomatología se relacionaría principalmente con la psicopatología del trastorno límite de personalidad (TLP) (Fruzzetti, Shenk \& Hoffman, 2005; Hankin, 2010). Además, se estima que un $32 \%$ de pacientes con TLP padece depresión (Widiger \& Trull, 1993). En el ámbito de la intervención se ha evidenciado la eficacia de las técnicas de psicología positiva para fomentar las emociones positivas, la calidad de vida, y la disminución del estado de ánimo negativo. Se considera que las intervencions positivas son eficaces para elevar los niveles de felicidad y que tienen un impacto significativo sobre la depresión (Seligman, Steen, Park \& Peterson, 2005; Vázquez, 2006; Vázquez, Hervás \& Ho, 2006). Un estudio 
realizado por Seligman, Rashid \& Parks (2006) evidenció que pacientes en terapia de grupo con psicoterapia positiva, reportaron mayor felicidad durante una semana y la sintomatología depresiva se redujo de forma significativa durante seis meses. Los resultados mostraron además que cuando la terapia positiva se personalizaba, ésta era más eficaz que la psicoterapia tradicional sola o con antidepresivos. Por otro lado, un meta-análisis realizado por Sin \& Lyubomirsky (2009), donde se analizaron 51 intervenciones basadas en psicología positiva, reveló que las intervenciones mejoraban de manera significativa el bienestar $(r=0,29)$ y dismuia las sintomatología depresiva $(r=0,31)$. Se realizó una comparativa entre grupos: (1) grupo control sin tratamiento, (2) tratamiento estándar y placebo; se encontró que las intervenciones positivas fueron más eficaces. A través de las actividades positivas el paciente crea conjunto de recursos de afrontamiento adaptativos que le permiten manejar situaciones o cogniciones negativas, disminuyendo los factores de riesgo de enfermedad metal (Layous, Chancellor \& Lyubomirsky 2014; Layous \& Lyubomirsky 2014). Por otro lado las aportaciones de Linehan (2003), con la terapia dialectico- conductual, suponen una contribución importante en la intervención con personas con TLP y por consiguiente para la intervención sobre aspectos específicos de regulación emocional. A través de su módulo de habilidades de regulación emocional, propone un modelo donde se enseña a identificar y etiquetar las emociones, reducir la vulnerabilidad negativa e incrementar acontecimientos positivos.

El objetivo de este trabajo es diseñar un protocolo de intervención adaptado a la problemática de la paciente para intervenir sobre problemas del estado de ánimo y rasgos de un possible TLP. El programa se compone de técnicas de regulación emocional y psicología positiva; se pretende someter a prueba su utilidad para la mejora de sintomatología. Asimismo se contempla el tratamiento como una intervención de carácter preventivo para futuras posibles psicopatologías. Previo al tratamiento se hipotetizó que las dos técnicas utilizadas darían lugar a cambios significativos en la sintomatología clínica.

\section{Método}

\section{Diseño}

Este estudio se basa en un diseño cuasi-experimental de estudio de caso único con pre y post evaluación.

\section{Participante}

María (nombre figurado), es una joven adulta de 23 años. Es la menor de dos hermanos y ambos viven en el domicilio del abuelo paterno.

Motivo de consulta: Acude demandando atención por problemas de autoestima, tristeza y celos. Cuenta que esto le genera un gran malestar y problemas en su estado de ánimo. Subraya antecedentes de depresión.

Descripción del caso: La paciente refiere que su vida ha estado marcada por fuertes acontecimientos estresantes, relacionados con su ambiente familiar. Fue abandonada en un centro de atención a menores, con cuatro años por su madre, la cual estaba diagnosticada de esquizofrenia. Tras un año en el centro, comenzó la convivencia con su padre. Con 12 años fallece su abuela paterna, ocasionando que ella asumiera el rol de ama de casa. Durante la misma franja temporal su padre inicia una nueva relación, que no fue aprobada por María. A 
la edad de 17 años su padre es diagnosticado de cáncer, asumiendo ella su cuidado y fallece al poco tiempo. Afirma que supuso el acontecimiento vital más importante, ocasionándole un periodo de depresión. Durante la misma época relata haber sufrido malos tratos por parte de su expareja. Actualmente vive con su abuelo paterno y su hermano y existe un conflictivo ambiente familiar. El hermano presenta antecedentes delictivos y consumo de sustancias. En la actualidad María refiere sentirse decaída, irritable, ansiosa, con sentimientos de inutilidad, y problemas de autoestima; comparándose continuamente con los demás. Afirma que sus problemas, están afectando a la relación con su pareja.

Anàlisis funcional: Se identifica la presencia de factores de riesgo (acontecimientos vitales estresantes) y esquemas cognitivos desadaptativos. Existe una retroalimentación entre las variables psicopatológicas y una vulnerabilidad psicológica generalizada que retroalimentan al mismo tiempo a la sintomatología depresiva. Sus dificultades en la regulación emocional juegan un papel importante en la fenomenología de los trastornos del estado de ánimo, contribuyendo al mantenimiento de los síntomas.

\section{Evaluación}

Se centró la evaluación en la medida de las siguientes variables: estado de ánimo; autoestima; personalidad; regulación emocional; expectativa y opinión sobre el tratamiento, y alianza terapéutica. Para ello se utilizó:

(1) Inventario de depresión de Beck (BDI-II). Auto-informe con 21 ítems de tipo Likert que proporciona una medida de la presencia y gravedad de la depresión (Beck, Steer \& Brown, 1996); validada en población española por Vázquez \& Sanz (1998).

(2) Escala de ansiedad estado/rasgo (STAI). Escala autoaplicada destinada a medir y evaluar el actual nivel de ansiedad y la predisposición a responder al estrés. Fue creada y validada para población española por Spielberger, Gorsuch \& Lushene (1982).

(3) Escala de autoestima de Rosenberg. Cuestionario auto-aplicado que evalúa la autoestima. Diseñada por Rosenberg (1965) y adaptada a población española por Echeburúa (1995). Consta de 10 ítems (5 planteados de forma positiva y los otros de forma negativa).

(4) Cuestionario multidimensional de personalidad (PAI). Cuestionario que permite la evaluación de la psicopatología de adultos. Tiene 22 escalas (4 de control, 11 clínicas, 5 de tratamiento y 2 de relaciones interpersonales). Fue diseñado por Morey (1991), y validado en población española por Ortiz-Tallo, Santamaría, Cardenal \& Sánchez (2011).

(5) Escala de dificultades en la regulación emocional (DERS). Escala autoaplicada, dirigida a evaluar y medir los procesos involucrados en la regulación afectiva. Fue diseñada por Gratz y Roemer (2004), y validada en población española por Hervás \& Jodar (2008).

(6) Entrevista neuropsiquiátrica internacional (M.I.N.I). Entrevista breve y estructurada, dirigida a explorar los principales trastornos psiquiátricos Sheehan et al., 1998).

(7) Expectativas y opinión del tratamiento. Escala que evalúa las expectativas del tratamiento antes de empezar, y el grado de satisfacción al finalizarlo, por parte del paciente (adaptado de Borkovec \& Nau, 1972). 
(8) Escala de gravedad valorada por el terapeuta. Adaptación de la escala Clinician Rating de Öst, Salkovskis \& Hellström (1991), realizada por Labpsitec. Está compuesta por 8 frases que indican la gravedad del paciente, y que el terapeuta debe valorar en el pre-tratamiento y post-tratamiento.

Asimismo se establecieron otras medidas a través de un registro semanal diseñado específicamente para este estudio. Se realizó un registro, que a través de la valoración diaria por parte de la paciente nos permitía evaluar: el grado de tristeza; el grado de aprecio por sí misma, y el grado de percepción del afrontamiento de los problemas. La evaluación se realizó semanalmente, todos los días, en un rango de 0-10.

\section{Diagnóstico}

Nos encontramos ante un perfil de personalidad, marcado por la desregulación emocional, la impulsividad, y la presencia de estrés, ansiedad y depresión. Presenta dificultades en el control de la ira, baja tolerancia a la frustración, sentimientos de tristeza y labilidad emocional. Se trata de una persona con rasgos de personalidad límite sin llegar a cumplir criterios diagnósticos. Como diagnóstico principal se obtiene un trastorno de depresión mayor con episodio único de tipo moderado 296.23 (F32.2) y en remisión total, con ansiedad moderada. Cabe añadir que pese a que en las evaluaciones de estado de ánimo ha puntuado alto, de acuerdo a los criterios diagnósticos del DSM-5, cumple criterios para un TDM moderado.

\section{Procedimiento}

Los resultados de la evaluación inicial aportaron información para el diseño de una intervención personalizada. Posteriormente se aplicó el tratamiento, y se realizó la evaluación post-tratamiento a fin de observar los cambios producidos en las variables de medida objetivo. La paciente contactó vía telefónica con el centro, se le explicaron los objetivos y características del estudio y se requirió el consentimiento informado de su participación voluntaria. El protocolo de intervención diseñado gira entorno a dos ejes principales: la regulación emocional y la psicología positiva. La duración total del tratamiento fue de 13 sesiones con una periodicidad semanal y una duración de 60 min. Está compuesto por cinco módulos: psicoeducación (2 sesiones), toma de conciencia (2 sesiones), regulación emocional (5 sesiones), psicología positiva (3 sesiones) y prevención de recaídas (1 sesión). Para el diseño del protocolo tomamos como referencia las aportaciones de Linehan (2003) para el tratamiento de personas con TLP, adaptando su módulo de regulación emocional a las características sintomatológicas de la paciente. El módulo de psicología positiva fue también una adaptación de las aportaciones sobre intervenciones positivas de Seligman (2002).

\section{Resultados}

Se realizó un análisis de la eficacia del tratamiento a través del estudio de las oscilaciones de las puntuaciones de cada instrumento de evaluación. Se compararon los resultados obtenidos en la evaluación inicial con los del final del tratamiento. Asimismo se analizó la evolución de la paciente a lo largo de todo el tratamiento atendiendo a las medidas de los registros semanales. En la Tabla 1 se muestra la evolución de la paciente, teniendo en cuenta las varia- 
bles de medida respecto al estado de ánimo (depresión y ansiedad), autoestima y regulación emocional. Los rasgos de personalidad es una variable que se tuvo en cuenta a la hora de la evaluación, sin embargo no se realizó una evaluación post-tratamiento ya que no fue el foco de la intervención. Nuestro objetivo fue trabajar sobre las variables que influyen en el estado de ánimo. En la evaluación pre-tratamiento la paciente presentaba una depresión grave y ansiedad estado y rasgo por encima de la media. Esto nos indica la presencia de ansiedad en el momento de la evaluación, así como su tendencia general a experimentar estados de ansiedad, existiendo pues, una relación con respecto a los niveles de intensidad en que la experimenta. Asimismo presenta una autoestima muy baja y dificultades de regulación emocional. Las puntuaciones en las tres primeras escalas del DERS (Confusión, Desatención y Rechazo) aluden a dificultades en los procesos de regulación. Las puntuaciones de Confusión y Rechazo se encuentran por encima de la media, siendo esta 7,9 y 14,9 respectivamente. La puntuación en Desatención se encuentra por debajo de la media $(9,6)$. Por su parte, las puntuaciones en Rechazo e Interferencia, son índices generales de desregulación emocional. Los resultados muestran puntuaciones por encima de la media española en ambas sub-escalas, siendo esta 16,5 y 10,2 respectivamente. Estos resultados se relacionan con la incapacidad percibida para el manejo de emociones y su influencia en la vida cotidiana.

Tras la evaluación post-tratamiento observamos una reducción significativa de la sintomatología. La puntuación total en la última evaluación, refleja una depresión mínima, autoestima media, y una reducción de la ansiedad, así como mejoras en su regulación emocional.

Las puntuaciones post-tratamiento en las sub-escalas del DERS, disminuyen ajustándose a la media. Por su parte se produce una mejora significativa en el manejo de la ansiedad, ajustándose tanto la ansiedad estado como la ansiedad rasgo a la media (atendiendo a la baremación española). Se observan, por tanto, cambios a nivel cuantitativo. No obstante, observamos puntuaciones muy polarizadas, disminuciones abultas que se relacionarían con su patrón de cambio emocional rápido y extremo, propio de su inestabilidad emocional. Seguidamente se muestra en la Figura 2 y Figura 3, la evolución a través de los registros semanales. La distribución de los módulos semanalmente fue: psicoeducación (sesiones 1 y 2), toma de conciencia (sesiones 3 y 4), regulación emocional (sesiones 5-9) y psicología positiva (sesiones 10-12). Cabe añadir que de la sesión 10 no pudieron recogerse datos debido a que la paciente no cumplimentó el registro.

Tabla 1

Resultados de la evaluación pre-tratamiento y post-tratamiento

\begin{tabular}{|c|c|c|c|c|c|}
\hline Variable & Test & \multicolumn{2}{|c|}{ Pre-Tratamiento } & \multicolumn{2}{|c|}{ Post-Tratamiento } \\
\hline Depresión & BDI-II & \multicolumn{2}{|c|}{$\begin{array}{l}\text { PT32 } \\
\text { Depresión grave }\end{array}$} & \multicolumn{2}{|c|}{$\begin{array}{l}\text { PT6 } \\
\text { Mínima depresión }\end{array}$} \\
\hline \multirow[t]{2}{*}{ Ansiedad } & STAI & Estado & Rasgo & Estado & Rasgo \\
\hline & & $\begin{array}{l}\text { PT34 } \\
\text { Centil } 80\end{array}$ & $\begin{array}{l}\text { PT44 } \\
\text { Centil } 96\end{array}$ & $\begin{array}{l}\text { PT7 } \\
\text { Centil } 5\end{array}$ & $\begin{array}{l}\text { PT14 } \\
\text { Centil } 15\end{array}$ \\
\hline Autoestima & Autoestima Rosenberg & $\begin{array}{l}\text { PT10 } \\
\text { Autoestim }\end{array}$ & & $\begin{array}{l}\text { PT26 } \\
\text { Autoesti }\end{array}$ & dia \\
\hline
\end{tabular}




\begin{tabular}{llll}
\hline Variable & Test & Pre-Tratamiento & Post-Tratamiento \\
\hline $\begin{array}{l}\text { Regulación Emoci- } \\
\text { onal }\end{array}$ & DERS & $\begin{array}{l}\text { Confusión: PT11 } \\
\text { Desatención: PT9 }\end{array}$ & $\begin{array}{l}\text { Confusión: PT7 } \\
\text { Desatención: PT18 }\end{array}$ \\
& & $\begin{array}{l}\text { Rechazo: PT22 } \\
\text { Descontrol: PT35 } \\
\text { Interferencia: PT19 }\end{array}$ & $\begin{array}{l}\text { Rechazo: PT7 } \\
\text { Descontrol: PT15 } \\
\text { Interferencia: PT7 }\end{array}$ \\
Gravedad & $\begin{array}{lll}\text { Escala de gravedad } \\
\text { Valorada por el terapeuta }\end{array}$ & $\begin{array}{l}\text { PT6 Grave con síntomas } \\
\text { incapacitantes en diferen- }\end{array}$ & $\begin{array}{l}\text { PT1 Leve los síntomas } \\
\text { pueden estar ocasional- } \\
\text { tes formas }\end{array}$ \\
& & & $\begin{array}{l}\text { mente presentes pero } \\
\text { apenas son percibidos } \\
\text { por el paciente }\end{array}$ \\
\hline
\end{tabular}

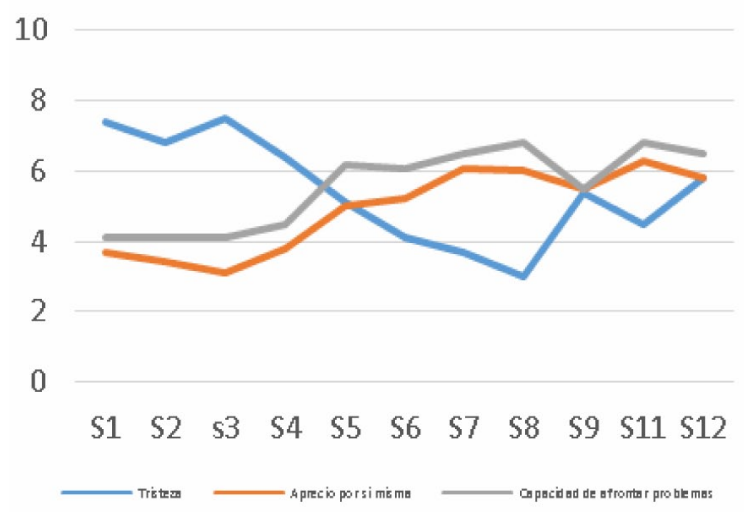

Figura 2. Tendencia en el tiempo del cambio de las variables

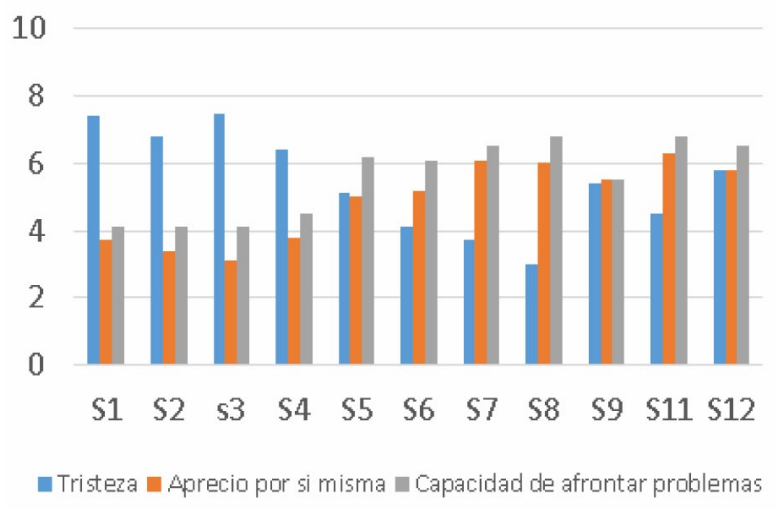

Figura 3. Comparación de los valores de las variables

Se observa como la tristeza presenta oscilaciones y que a partir de la primera sesión de toma de conciencia comienza a disminuir. Durante las sesiones de regulación emocional se mantiene relativamente estable llegando a una puntuación de 3 respecto al 7,5 obtenido en la primera semana. Sin embargo, a partir de la semana 8 (S8) los resultados muestran un aumento de los niveles de tristeza obteniendo una puntuación de 5,4 en la semana. Estos resultados se deben a circunstancias externas a la intervención, acontecimientos personales que acaecieron en torno a la vida de la paciente: su mascota, (siendo uno de sus pilares más importantes) enfermó, por lo que los niveles de tristeza aumentaron notablemente.

No obstante, pese al aumento en las últimas semanas, los niveles de tristeza presentan cambios positivos, que se observan además durante las sesiones de intervención. La paciente manifiesta mejoras en las relaciones interpersonales, mejoras en el ambiente familiar, sentirse más animada y afirma que los cambios son reconocidos por las personas que se encuentran a su alrededor (amigos y pareja). El resto de variables sufre también modificaciones significativas: la capacidad de afrontamiento de problemas aumenta tras las estrategias de regulación emocional, donde se le enseña a identificar emociones, aceptarlas y regularlas. Del mismo modo, las técnicas de psicología positiva parecen ejercer también una gran influencia en la visión positiva de los problemas y de sí misma. Asimismo, y pese a que estas variables descienden en la última semana, viéndose influidas por la muerte de su mascota, llama 
la atención: el aumento de su capacidad para afrontar situaciones difíciles con respecto al principio de la intervención, la disminución de la tristeza y el incremento en su autoestima. La Figura 3 es un gráfico de columnas agrupadas que se utiliza para comparar valores entre algunas categorías, muestra una comparativa en relación a la evolución de cada una de las variables. Se observa como la tendencia a la baja de la tristeza se relaciona con el aumento de la visión positiva de sí misma y la capacidad de afrontamiento de los problemas. Se infiere por tanto, la influencia de unas variables sobre otras, así como la estrecha relación de la capacidad de afrontar problemas con las mejoras en autoestima, aprecio y sentimientos de valía personal. Los resultados post-tratamiento sobre la opinión de la intervención se relacionan con los resultados pre-tratamiento respecto a las expectativas del mismo por parte de la paciente. Asimismo, se obtuvo una buena puntuación en alianza terapéutica tanto pre como post-tratamiento.

\section{Discusión y conclusiones}

En primer lugar y atendiendo a los resultados de la evaluación pre-tratamiento y al análisis funcional de la paciente observamos una fuerte vulnerabilidad asociada a un conjunto significativo de factores de riesgo. Tal y como afirman y demuestran otros estudios, los acontecimientos vitales estresantes suponen factores de riesgo para el desarrollo de depresión: esto se puede ver potenciado por características de personalidad desadaptativas (p. ej., rasgos latentes de personalidad limite), que ocasionan dificultades en la regulación emocional, alteraciones en del sistema emocional, e inestabilidad afectiva (Fruzzetti et al., 2005; Hankin, 2010; Lloyd et al., 2015). Se observa un cambio en los valores del BDI-II, pasando de una depresión grave a mínima. Estos resultados se relacionan con lo identificado en otros estudios sobre la eficacia de la psicología positiva sobre la disminución de la sintomatología depresiva (Seligman et al., 2005; Vázquez, 2006; Vázquez et al., 2006). Por ejemplo, el estudio llevado a cabo por Seligman et al. (2006), mostró como la psicoterapia basada en técnicas de psicología positiva fue altamente eficaz en la reducción de los síntomas depresivos y que además lo era aún más de forma individualizada. Por otro lado los resultados proporcionados por el meta-análisis de Sin \& Lyubomirsky (2009), mostraron también una reducción significativa de los síntomas de depresión y una mejora del bienestar emocional, tras el análisis de 51 intervenciones basadas en psicología positiva. Por su parte la regulación emocional de la paciente se ha visto también mejorada, obteniendo cambios positivos y evidenciando mejoras en la solución de problemas, aceptación, y gestión de emociones. Estos resultados, se relacionan con los beneficios reportados por Linehan (2003), respecto a enseñar habilidades de regulación emocional para entender, conocer y aceptar las propias emociones, reducir la vulnerabilidad y sufrimiento emocional y potenciar las experiencias positivas de la vida diaria. Realizando un análisis general de los resultados obtenidos, se concluye por un lado que el aumento en la percepción de capacidad de solución de problemas por parte de la paciente, presenta un aumento significativo durante el módulo de regulación emocional. Estos resultados nos hacen suponer la importancia e influencia de la gestión emocional adaptativa, como base para el aprendizaje y desarrollo de habilidades funcionales de resolución de conflictos. Del mismo modo otra conclusión extraída de dichos resultados es la eficacia de la psicología positiva, no solo para el bienestar emocional sino también para la autoestima y la visión positiva de los problemas. Los resultados de los registros semanales muestran un aumento de esta variable coincidiendo con las últimas sesiones y relacionándose al mismo tiempo con la mejora en la solución de problemas. Asimismo es importante tener en cuenta que pese a que en un principio la muerte de su mascota pueda haberse considerado como una interferencia en la intervención, finalmente 
analizando los resultados, observamos que ha sido una condición externa que nos ha ayudado a comprobar que efectivamente ha mejorado sus competencias y habilidades personales respecto a su capacidad para gestionar las emociones y hacer frente a situaciones adversas; aumentando su autoeficacia y sentimientos de valía personal. No obstante es importante tener en cuenta que pese a ello, la paciente presenta una elevada vulnerabilidad a experimentar los acontecimientos negativos con una mayor intensidad emocional, debida principalmente a los rasgos de personalidad que la caracterizan. Es por ello por lo que observamos niveles de tristeza muy intensos tras la muerte de su mascota, que se contrastaron y relacionaron con las manifestaciones en sesión de la paciente.

\section{Limitaciones y sugerencias}

El diseño se ciñe a la intervención individual, por lo tanto, estos resultados se extrapolan solo al efecto particular sobre el caso en cuestión. Cabe mencionar que la paciente llegaba tarde en numerosas ocasiones, lo que limitaba el tiempo de intervención. Esto podría relacionarse con el carácter gratuito de la intervención, ocasionando así percepción de menor implicación y responsabilidad. En la misma línea la paciente no cumplimentó los registros correspondientes a la semana 10, por lo que no hemos podido realizar un análisis de los mismos. Otras interferencias destacadas son la no realización de todas las tareas y la cancelación de diferentes sesiones que supusieron alargar el tratamiento. No obstante, estos comportamientos se relacionarían de forma significativa con su patrón de personalidad. El estudio presentado puede tomarse como una investigación preliminar e individual, que podría ponerse en práctica a nivel grupal. Asimismo, se podrían llevar a cabo futuras investigaciones con grupo control, a fin de realizar una comparativa de la eficacia conjunta de estas técnicas respecto a su uso de forma independiente.

\section{Referencias bibliográficas}

Beck, A. T., Steer, R. A. \& Brown, G. K. (1996). Manual for the Beck Depression Inventory-II. San Antonio, TX: Psychological Corporation.

Borkovec, T. D. \& Nau, S. D. (1972). Credibility of analogue therapy rationales. Journal of Behavior Therapy and Experimental Psychiatry 3, 257-260.

Echeburúa, E. (1995). Evaluación y tratamiento de la fobia social. Barcelona: Martínez Roca.

Fruzzetti, A. E., Shenk, C., y Hoffman, P. D. (2005). Family interaction and the development of borderline personality disorder: A transactional model. Development and Psychopathology, 17(04), 1007-1030.

Gratz, K. L. \& Roemer, L. (2004). Multidimensional assessment of emotion regulation and deregulation: Development, factor structure, and initial validation of the difficulties in emotion regulation scale. Journal of Psychopathology and Behavioral assessment, 26(1), 41-54.

Hankin, B. L. (2010). Personality and depressive symptoms: Stress generation and cognitive vulnerabilities to depression in a prospective daily diary study. Journal of Social and Clinical Psychology, 29(4), 369-401.

Hervás, G. \& Jódar, R. (2008). Adaptación al castellano de la Escala de Dificultades en la Regulación Emocional. Clínica y Salud, 19(2), 139-156. 
Hervás, G. \& Vázquez, C. (2006). La regulación afectiva: Modelos, investigación e implicaciones para la salud mental y física. Revista de Psicología General y Aplicada, 59(1-2), 9-36.

Layous, K., Chancellor, J. \& Lyubomirsky S. (2014). Positive activities as protective factors against mental health conditions. Journal of Abnormal Psychology, 123(1), 3-12.

Layous, K. \& Lyubomirsky S. (2014). The how, why what, when, and who of happiness: Mechanisms underlying the success of positive activity interventions. Positive emotion: Integrating the light sides and dark sides, 473-495.

Linehan, M. (2003). Manual de tratamiento de los trastornos de personalidad límite. Madrid: Paidós.

Lloyd, C., Klinteberg, B. \& DeMarinis, V. (2016). Emotion regulation and existential meaningmaking in young women with mental ill-health concerns-a qualitative study. International Journal of Psychology and Behavioral Sciences, 1(1), 1-11.

Morey L. C. (1991). The Personality Assessment Inventory: Professional manual. Odessa. FL: Psychological Assessment Resources.

Ortiz-Tallo, M., Santamaría, P., Cardenal, V. \& Sánchez, M. P. (2011). Adaptación española del Inventario de Evaluación de la Personalidad (PAI). Madrid: Ediciones TEA.

Öst, L. G., Salkovskis, P. \& Hellström, K. (1991). One-session therapist directed exposure vs. self-exposure in the treatment of spider phobia. Behavior Therapy, 22(3), 407-422.

Rosenberg, M. (1965). Society and the adolescent self-image. Princeton, N J: Princeton University.

Sanz, J. \& Vázquez, C. (1998). Fiabilidad, validez y datos normativos del Inventario para la Depresión de Beck. Psicothema, 10(2), 303-318.

Seligman, M. E. (2002). Positive psychology positive prevention and positive therapy. Handbook of Positive Psychology, 2, 3-12.

Seligman, M. E., Rashid, T., y Parks, A. C. (2006). Positive psychotherapy. American Psychologist, 61(8), 774-788.

Seligman, M. E., Steen, T. A., Park, N. \& Peterson, C. (2005). Positive psychology progress: Empirical validation of interventions. American Psychologist, 60(5), 410-421.

Sheehan, D. V., Janavs, J., Baker, R., Harnett-Sheehan, K., Knapp, E., Sheehan, M. \& Bonora, L. I. (1998). MINI-Mini International neuropsychiatric interview-english version 5.0. 0-DSM-IV. Journal of Clinical Psychiatry, 59, 34-57.

Sin, N. L. \& Lyubomirsky S. (2009). Enhancing well-being and alleviating depressive symptoms with positive psychology interventions: A practice-friendly meta-analysis. Journal of Clinical Psychology, 65(5), 467-487.

Spielberger, C. D., Gorsuch, R. L. \& Lushene, R. (1982). Manual del Cuestionario de Ansiedad Estado/Rasgo (STAl). Madrid, España: TEA Ediciones

Vázquez, C. (2006). La psicología positiva en perspectiva. Papeles del Psicólogo, 27(1), 1-2.

Vázquez, C., Hervás, G. \& Ho, S. (2006). Intervenciones clínicas basadas en la psicología positiva: fundamentos y aplicaciones. Psicología Conductual, 14(3), 401-432.

Widiger, T. A. \& Trull, T. J. (1993). Borderline and narcissistic personality disorders. En P. B. Sutker \& H. E. Adams (dirs.), Comprehensive handbook of psychopathology (pp. 371394). Nueva York: Plenum. 